\title{
Effect of Antibiosis on Antagonist Dose-Plant Disease Response Relationships for the Biological Control of Crown Gall of Tomato and Cherry
}

\author{
K. B. Johnson and J. A. DiLeone
}

First and second author: Department of Botany and Plant Pathology, Oregon State University, Corvallis 97331-2902; and second author: USDA-ARS Horticultural Crops Research Laboratory, Corvallis, OR 97330.

Accepted for publication 2 July 1999.

\begin{abstract}
Johnson, K. B., and DiLeone, J. A. 1999. Effect of antibiosis on antagonist dose-plant disease response relationships for the biological control of crown gall of tomato and cherry. Phytopathology 89:974-980.

The crown gall pathosystem was used to evaluate a model that describes the dose-response relationship between biological control agents and plant pathogens. The model predicts that this relationship can become asymptotic, such that increased antagonist doses cannot compensate for deficiencies in disease suppression. Wounded roots of tomato (Lycopersicon esculentum) and cherry (Prunus mahaleb) plants were dipped into different concentrations of the biological control organism Agrobacterium radiobacter strain K84 prior to inoculation with the pathogen A. tumefaciens. Pathogen strains sensitive or resistant to the antibiotic agrocin 84 were used, and for tomato experiments, a derivative of A. radiobacter strain $\mathrm{K} 84$ that does not produce agrocin 84 also was included as an experimental treatment. As predicted by the dose-response model, the amount of disease suppression
\end{abstract}

ABSTRACT

Biological control of plant disease commonly requires an inundative (massive) introduction of a microbial antagonist to obtain disease suppression. This requirement for a large initial dose of the antagonist implies that the amount of the microbe immediately available to interact with the pathogen is related to the degree of suppression obtained. A model developed to describe the relationship between pathogen dose and disease response was extended recently to the relationship between the size of an antagonist introduction and its resulting effect on the pathogen population and plant disease response (9). This model has described observed antagonist dose-plant disease response relationships, but to do so required inclusion of an asymptote (refuge) parameter that defined a proportion of a pathogen population that could not be impacted by an antagonist introduction, even at the highest antagonist doses. Expectations derived from this model showed that the magnitude of the asymptote greatly influences the amount of disease suppression achievable by an antagonist.

Several empirical studies $(15,18,19)$ have supported the concepts proposed within the antagonist dose-plant disease response model and shown that the magnitude of the asymptote can be affected by the antagonist strain within specific pathosystems. In these studies, dose-response models for effective strains had relatively large values of the parameter $A$ (e.g., 0.87 to 0.94 ), for which the value of $A$ subtracted from 1.0 is an estimate of the proportion of pathogen propagules that are not susceptible to suppression by an antagonist (this proportion of the pathogen population exists within a refuge). In contrast, dose-response models for ineffective antagonists typically had smaller estimates of $A$ (e.g., 0.00 to 0.67 ) and, consequently,

Corresponding author: K. B. Johnson; E-mail address: johnsonk@bcc.orst.edu

Publication no. P-1999-0812-01R

(c) 1999 The American Phytopathological Society per unit of antagonist decreased with increasing antagonist dose and became asymptotic at high antagonist densities. Control of crown gall of tomato was nearly complete with the combination of $A$. radiobacter $\mathrm{K} 84$ and an agrocin 84-sensitive strain of A. tumefaciens. Pathogen resistance to agrocin 84 or lack of agrocin 84 production by $A$. radiobacter resulted in antagonist dose-crown gall incidence relationships that were apparently asymptotic at levels of control significantly less than $100 \%$. For field-grown cherry, similar dose-response relationships were observed with higher asymptotic levels of disease suppression obtained when trees were inoculated with an agrocin 84-sensitive A. tumefaciens strain compared with an agrocin 84-resistant pathogen strain. The differences among bacterial strain combinations in the magnitude of the asymptote defined by the dose-response relationships suggest that $A$. radiobacter impacts a smaller proportion of the pathogen population when the activity of agrocin 84 is muted.

Additional keywords: plant disease epidemiology. a larger refuge size. The magnitude of the asymptote parameter appears to be an important regulator of the efficacy of biocontrol, but little is known as to how this parameter is affected by the various factors that operate in an effective antagonistic interaction. We have speculated $(9,10)$ that mechanisms of biocontrol could affect the value of the asymptote parameter in a modeled antagonist dose-plant disease response relationship. To our knowledge, however, the hypothesis that a factor known to affect the success of biological control does so principally by changing the size of the asymptotic refuge has not been tested directly.

Crown gall, caused by the soilborne bacterium Agrobacterium tumefaciens, is a disease for which biological control has been implemented commercially $(12,16,17)$. In plant nurseries, biocontrol is achieved when wounds on root-pruned plants are inoculated inundatively with a suspension of the bacterial antagonist $A$. radiobacter strain K84 (K84) before exposure to the pathogen (17). Most literature indicates that K84 is effective, at least in part, because of the in situ production of agrocin 84 , an antibiotic with specific toxicity against sensitive strains of A tumefaciens $(6,7$, 12). The significance of agrocin 84 in biocontrol of crown gall has been evaluated by comparing the efficacy of wild-type K84 with derived strains that lack the ability to produce agrocin 84 and by contrasting the efficacy of K84 when disease was incited by agrocin 84-sensitive or agrocin 84-resistant strains of A. tumefaciens. These experiments typically show that disease control is diminished when either agrocin 84-deficient $A$. radiobacter $(4,13)$ or agrocin 84-resistant A. tumefaciens is a component of the antagonist-pathogen interaction $(3,12,13)$. With regard to dose-response relationships, however, it is not known whether increasing the dose of K84 can make up for diminished control or if, by muting the mechanism of antibiosis, the size of the pathogen refuge is increased. For the latter alternative, the model predicts that increases in antagonist density will cause only negligible increases in disease suppression (9). 
The objective of this study was to test the following hypotheses: that the antagonist dose-plant disease response relationship in the crown gall pathosystem can be described by the model we proposed, that this description will define a pathogen refuge, and that the mechanism of antibiosis will affect refuge size.

\section{MATERIALS AND METHODS}

Dose-response model. The antagonist dose-plant disease response model was published previously (9) and is based on a common relationship between pathogen dose and disease response $(1,8,21)$

$$
y=1-\exp (-k x)
$$

in which $y$ is the proportion of plant units that become diseased, $x$ is the inoculum density of a pathogen, and $k$ is a constant that represents the efficiency at which pathogen propagules infect plant units. Analogously, the effect of a biological antagonist on a pathogen population is represented by

$$
x_{i} / x=A[1-\exp (-c z)]
$$

in which $z$ is antagonist density, $c$ is the efficiency of an antagonist propagule, and $x_{i} / x$ is the proportion of effective pathogen units that become ineffective as a result of the antagonist introduction. The asymptote variable, $A$, represents the maximum proportion of effective pathogen units that can be rendered ineffective by the antagonist. Conversely, the value $1-A$ is the proportion of pathogen propagules that exist within a refuge and, thus, cannot be rendered ineffective by the antagonist introduction. The combination of equations 1 and 2

$$
y=1-\exp (-k x\{[1-A]+A[\exp (-c z)]\})
$$

links incidence of disease with antagonist density. When disease incidence is observed over a range of antagonist doses, equation 3 provides a framework for estimation of $A, c$, and $k x$ with statistical procedures.

Bacterial strains. Antagonist strains used in experiments were A. radiobacter strains K84 and K434 (strains JL5024 and JL5018, respectively, obtained from J. Loper, USDA-ARS Horticultural Crops Research Lab, Corvallis, OR). K84 produces agrocin 84 (12), whereas K434 is a derivative of K84 cured of plasmid pAgK84 and, thus, does not produce the antibiotic (14). For tomato experiments, A. tumefaciens biovar 1 strain C58, sensitive to agrocin 84, was used (obtained from L. Moore, Department of Botany and Plant Pathology, Oregon State University, Corvallis). A spontaneous, agrocin 84-resistant mutant of C58, strain C58-R124, was also used (obtained from D. Cooksey, Department of Plant Pathology, University of California, Riverside) (3). For a cherry experiment, biovar 2 strains of $A$. tumefaciens, $\mathrm{K} 27 \mathrm{Rf}^{\mathrm{T}}$, sensitive to agrocin 84 and resistant to rifampicin, and $\mathrm{B} 49 \mathrm{c} \mathrm{Rf}^{\mathrm{T}}$, resistant to agrocin 84 and rifampicin, were used (obtained from L. Moore)

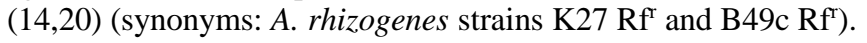

Inoculum preparation. Agrobacterium strains were cultured for 5 days at 20 to $25^{\circ} \mathrm{C}$ on mannitol-glutamate agar (11) amended with $1 \mathrm{~g}$ of yeast extract per liter (MGY). Bacteria were scraped from the surface of the agar and suspended in $10 \mathrm{mM}$ phosphate buffer $(\mathrm{pH} 7)$. Cell densities of antagonist strains were adjusted to $9 \times 10^{8}$ and $5 \times$ $10^{8} \mathrm{CFU} / \mathrm{ml}$ in the tomato and cherry experiments, respectively, with aid of a spectrophotometer (600 nm, model Spec 20; Bausch and Lomb Optical Co., Rochester, NY); pathogen strains were adjusted to $1 \times 10^{8} \mathrm{CFU} / \mathrm{ml}$. These concentrations were verified in each experiment by spreading dilutions of the suspensions on MGY. The ranges of antagonist and pathogen concentrations used to treat plants were prepared by diluting the highest concentration with buffer.

Tomato experiments. In the greenhouse, tomato seeds (Lycopersicon esculentum Mill. cv. Bonny Best) were sown in flats that contained a 1:1 mixture of peat moss (Canadian sphagnum; Lakeland Ltd., Edmonton, Alberta, Canada) and propagation grade perlite
(Supreme Perlite Co., Portland, OR) and watered routinely. After emergence, seedlings were drenched twice a week with a soluble fertilizer (Plantex 20-20-20 [N-P-K], 200 ppm; PlantCo Inc., Brampton, Ontario, Canada). At 3 weeks after emergence, tomato plants were uprooted and a third of the root tissue was removed by pruning. Root-pruned plants were soaked in an antagonist treatment suspension for $15 \mathrm{~min}$. Antagonist treatments were K84 or K434 at each of the following concentrations: $0,5 \times 10^{6}, 5 \times 10^{7}, 1 \times 10^{8}, 2.5 \times 10^{8}$, $5 \times 10^{8}, 7 \times 10^{8}$, and $9 \times 10^{8} \mathrm{CFU} / \mathrm{ml}$. Dilution plating was used to verify initial doses deposited onto root surfaces. The most concentrated treatment suspension, $9 \times 10^{8} \mathrm{CFU} / \mathrm{ml}$, resulted in an initial deposition of $2 \times 10^{5} \mathrm{CFU} / \mathrm{cm}$ of root with initial depositions for other treatments maintaining their expected proportionality. After a 5 -min drying, the plants were soaked in a suspension $\left(1 \times 10^{7}\right.$ $\mathrm{CFU} / \mathrm{ml}$ ) of C58 or C58-R124 for $15 \mathrm{~min}$. After another 5-min drying period, the plants were transplanted into pots $(10 \times 10 \times 8 \mathrm{~cm})$ that contained washed sand (four plants per pot), watered, and placed in a shaded humidity chamber for $48 \mathrm{~h}$ to minimize transplant shock. Potted plants were treated with a slow-release fertilizer (Nutricote 14-14-14 [N-P-K], 5 g per pot; PlantCo Inc.) and grown in the greenhouse for 5 weeks. At the end of this period, the incidence of crown gall was determined by inspecting the root systems for the incidence of galls. Experimental design was a randomized complete block with five replications blocked over time. Twenty plants per replication were inoculated with each combination of antagonist strain $\times$ antagonist concentration $\times$ pathogen strain. The experiment was conducted twice, with individual replications inoculated between 10 July and 27 August 1996 in the first experiment and between 4 September and 11 October 1996 in the second experiment.

Cherry experiments. In April 1997, 1-year-old seedlings of mahaleb cherry (Prunus mahaleb L.) were obtained in a dormant, bare-root condition from a commercial nursery. Cherry seedlings were held in cold storage $\left(4^{\circ} \mathrm{C}\right)$ until a few days before inoculation, when they were tied in bundles of 33 plants and acclimated to outdoor conditions. At inoculation (1 May), about one half of the taproot was removed from each seedling to create a fresh wound. Immediately after root pruning, the seedlings were soaked in a suspension of K84 for $15 \mathrm{~min}$. Suspensions of K84 were prepared at the following concentrations: $0,5 \times 10^{6}, 5 \times 10^{7}, 1 \times 10^{8}, 2.5 \times 10^{8}$, and $5 \times 10^{8}$ CFU/ml. A 1-h drying period followed the antagonist treatment, after which the seedlings were soaked in one of two concentrations of $\mathrm{K} 27 \mathrm{Rf}^{\mathrm{T}}$ or $\mathrm{B} 49 \mathrm{c} \mathrm{R \textrm {R } ^ { \mathrm { T } }}$ for $15 \mathrm{~min}$. Pathogen concentrations were $1 \times 10^{7}$ and $1 \times 10^{8} \mathrm{CFU} / \mathrm{ml}$. Inoculated seedlings were held in moist sawdust overnight and planted the next day into a Chehalis loam soil located at the Oregon State University, Botany and Plant Pathology Field Laboratory near Corvallis. Plots were irrigated routinely by overhead sprinkler and fertilized once at 3 to 4 weeks after planting by banding in the row 33-0-0 (N-P-K) at a rate of $55 \mathrm{~kg} / \mathrm{ha}$. Disease was assessed in October by uprooting the trees and examining the taproot for incidence of crown gall. Experimental design was a randomized block with six replications; 33 seedlings per replication were inoculated with each combination of antagonist concentration $\times$ pathogen strain $\times$ pathogen concentration. Attrition of cherry seedlings after planting averaged $6 \%$ and was not affected by any of the bacterial treatments.

Data analysis. Parameter estimates for the dose-response model were obtained for each antagonist $\times$ pathogen strain combination following procedures outlined previously (9). Crown gall incidence data for each replication $(y)$ were first transformed $(-\ln [1-y])$ to correct for multiple infection (8) and then averaged across replication. For each pathogen treatment (strain or concentration), the initial estimate for $k x$ was taken as the mean value of $-\ln (1-y)$ when the concentration of the antagonist, $z$, was equal to 0.0. An initial estimate for $A$ was calculated by

$$
A=1+[-\ln (1-y) / k x]+0.001
$$

using the lowest mean value of $-\ln (1-y)$ obtained over each series of concentrations of an antagonist strain. Replicate means for 
$-\ln (1-y)$ were then backtransformed and, with initial estimates of $k x$ and $A$, retransformed

$$
-\ln (-\ln \{[1-y] / \exp [-k x(1-A)]\})
$$

Values obtained with equation 5 were regressed linearly on antagonist concentration $(z)$ to provide a first estimate of the antagonist efficiency parameter, $c$.

Final estimates and asymptotic standard errors of $k x, A$, and $c$ were obtained using a nonlinear regression procedure (PROC NLIN, Secant method, SAS System for Windows release 6.12; SAS Institute, Cary, NC), which provided iterative solutions to equation 3 and to a transformed version of equation 3

$$
-\ln (1-y)=k x[(1-A)+A \cdot \exp (-c z)]
$$

Means of $-\ln (1-y)$ averaged across replication were used as the input data for regressions based on equation 6 , whereas disease incidence values for individual replicates were used for nonlinear regressions based on equation 3. If a regression solution was obtained for an antagonist $\times$ pathogen strain combination, residuals were plotted against antagonist density to evaluate the acceptability of the fitted models (2). The null hypotheses that $A=1.0$ (a pathogen refuge does not exist) and $c=0.0$ (antagonist efficiency was low) were evaluated for each modeled antagonist $\times$ pathogen strain combination. Confidence intervals of $95 \%$ were obtained for estimates of $k x, A$, and $c$. Confidence intervals for parameter estimates from models in which observable activity of agrocin 84 was expected were compared with parameter estimates in which agrocin 84 activity was not expected, and vice versa. Parameters were considered significantly different $(P \leq 0.05)$ if neither interval overlapped the parameter estimate of the other.

\section{RESULTS}

Tomato experiments. Crown gall incited by C58 and C58-R124 was apparent on tomato roots at 5 weeks after inoculation. For plants inoculated with a pathogen strain but not treated with $A$. radiobacter, incidence of galled plants in experiment 1 averaged $86 \pm 4$ (standard error) and $89 \pm 5 \%$ for C58 and C58-R124, respectively. In experiment 2 , incidence of galled plants was $72 \pm 5$ and $76 \pm 6 \%$ for C58 and C58-R124, respectively. On plants treated with a high concentration $\left(\geq 2.5 \times 10^{8} \mathrm{CFU} / \mathrm{ml}\right)$ of $\mathrm{K} 84$ followed by the agrocin 84-sensitive strain C58, incidence of crown gall averaged $10 \pm 5$ and $9 \pm 3 \%$ for experiments 1 and 2, respectively. In contrast, crown gall incidence on plants that were treated with a high concentration of K84 prior to inoculation with the agrocin 84-resistant strain C58-R124 averaged $80 \pm 7$ and $59 \pm 8 \%$ for experiments 1 and 2, respectively. Similarly, incidence of crown gall on plants treated with a high concentration of the agrocin 84-deficient strain K434 prior to inoculation with C58 or C58-R124 averaged $61 \pm 12$ and $71 \pm 9 \%$, respectively, for experiment 1 , and $47 \pm$ 5 and $46 \pm 8 \%$, respectively, for experiment 2 .

Initial estimates of the asymptote parameter, $A$, varied among antagonist $\times$ pathogen strain combinations (Table 1). Values of 0.99 (experiment 1) and 0.97 (experiment 2) were obtained for the combination of $\mathrm{K} 84$ with $\mathrm{C} 58$, whereas values ranging from 0.46 to 0.73 were obtained for the strain combinations of K84 with C58R124 and K434 with C58 or C58-R124. Initial estimates of the efficiency parameter, $c$, were relatively consistent among strain combinations, with all values in the range of 1.5 to $7.5 \times 10^{-9}$ (Table 1). Additionally, for all initial estimates of $c$, except for the combination of K84 with C58-R124 in experiment 1, a linear trend was apparent in the regression of $-\ln (-\ln \{[1-y] / \exp [-k x(1-A)]\})$ on antagonist density (z) (Fig. 1, results for experiment 2 ). For regression analyses based on equation 6 , models were fit to six of the eight data sets but not for the combination of K84 with C58R124 in either experiment 1 or 2 (Table 1). Final estimates of $A$ obtained after nonlinear regression of $-\ln (1-y)$ on antagonist concentration were, on average, $15 \%$ smaller than initial estimates, and several final estimates of $c$ varied by more than a log unit from the initial estimate. Regression analyses based on equation 3 resulted in fits to seven of eight data sets; the exception was K434 with C58-R124 in experiment 1 . Estimates of $A$ and $c$ based on equation 3 were generally similar to those obtained with equation 6 , but estimates of $k x$ based on equation 3 were 3 to $13 \%$ smaller.

For data sets in which the regression procedure was able to provide a solution, equations 6 (Fig. 2) and 3 (Fig. 3) appeared to represent the observed incidence of crown gall of tomato as a function of initial antagonist concentration. This conclusion also was supported by the patterns of residuals derived from the regression analyses (data not shown). For both tomato experiments, the null hypothesis that $A=1.0$ (all pathogen propagules are susceptible to the antagonist) could not be rejected for the strain combination of K84 with C58 in either experiment $(P>0.05)$ when fit to equation 6 (Table 1). This hypothesis was rejected, however, for K84 with C58 when the data were fit to equation 3 , owing to smaller estimates for the asymptotic standard error of $A$. The null hypothesis, $A=1.0$, also was rejected for all models based on strain combinations in which the activity of agrocin 84 was not expected to influence disease control (K84 with C58-R124, and K434 with C58

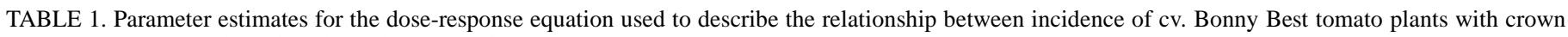

\begin{tabular}{|c|c|c|c|c|c|c|c|c|c|c|}
\hline \multirow[b]{2}{*}{ Experiment $^{\mathrm{a}}$} & \multirow{2}{*}{$\begin{array}{l}\text { Antagonist/pathogen } \\
\text { strain combination }^{\mathrm{b}}\end{array}$} & \multicolumn{3}{|c|}{ Initial estimates $^{c}$} & \multicolumn{3}{|c|}{ Final estimates ${ }^{\mathrm{d}}$ for equation $6^{\mathrm{e}}$} & \multicolumn{3}{|c|}{ Final estimates for equation $3^{\mathrm{f}}$} \\
\hline & & $k x$ & $A$ & $c$ & $k x$ & $A$ & $c$ & $k x$ & $A$ & $c$ \\
\hline 1 & K84/C58 & 2.09 & 0.99 & $7.48 \mathrm{E}-9$ & $2.12 \pm 0.15$ & $0.95 \pm 0.05$ & $2.31 \mathrm{E}-8 \pm 6.11 \mathrm{E}-9 * * \mathrm{~g}$ & $1.85 \pm 0.21$ & $0.94 \pm 0.02 * \mathrm{~h}$ & $2.32 \mathrm{E}-8 \pm 3.82 \mathrm{E}-9 * *$ \\
\hline 1 & K434/C58 & 2.09 & 0.73 & $5.78 \mathrm{E}-9$ & $2.36 \pm 0.28$ & $0.51 \pm 0.07 *$ & $7.47 \mathrm{E}-8 \pm 7.90 \mathrm{E}-8$ & $2.05 \pm 0.50$ & $0.53 \pm 0.12 *$ & $1.27 \mathrm{E}-7 \pm 1.76 \mathrm{E}-7$ \\
\hline 1 & K84/C58-R124 & 2.62 & 0.46 & $1.54 \mathrm{E}-9$ & $\ldots{ }^{\mathrm{i}}$ & $\ldots$ & & $2.06 \pm 0.31$ & $0.25 \pm 0.12 *$ & $1.37 \mathrm{E}-7 \pm 2.97 \mathrm{E}-7$ \\
\hline 1 & K434/C58-R124 & 2.62 & 0.57 & $5.08 \mathrm{E}-9$ & $2.57 \pm 0.21$ & $0.47 \pm 0.08 *$ & $8.36 \mathrm{E}-9 \pm 5.13 \mathrm{E}-9$ & & & \\
\hline 2 & K84/C58 & 1.33 & 0.97 & $5.89 \mathrm{E}-9$ & $1.15 \pm 0.14$ & $0.92 \pm 0.09$ & $1.14 \mathrm{E}-8 \pm 5.18 \mathrm{E}-9 * *$ & $1.12 \pm 0.10$ & $0.92 \pm 0.03 *$ & $1.13 \mathrm{E}-8 \pm 2.39 \mathrm{E}-9 * *$ \\
\hline 2 & K434/C58 & 1.33 & 0.62 & 4.17E-9 & $1.42 \pm 0.10$ & $0.46 \pm 0.04 *$ & $1.03 \mathrm{E}-7 \pm 5.47 \mathrm{E}-8$ & $1.34 \pm 0.13$ & $0.49 \pm 0.06^{*}$ & $9.80 \mathrm{E}-8 \pm 8.27 \mathrm{E}-8$ \\
\hline 2 & K84/C58-R124 & 1.53 & 0.46 & $3.85 \mathrm{E}-9$ & $\ldots$ & $\ldots$ & $\ldots$ & $1.13 \pm 0.09$ & $0.41 \pm 0.24^{*}$ & $1.49 \mathrm{E}-8 \pm 4.16 \mathrm{E}-9$ \\
\hline 2 & K434/C58-R124 & 1.53 & 0.60 & $3.80 \mathrm{E}-9$ & $1.43 \pm 0.07$ & $0.56 \pm 0.03^{*}$ & $4.41 \mathrm{E}-7 \pm 1.72 \mathrm{E}-7$ & $1.35 \pm 0.15$ & $0.53 \pm 0.06^{*}$ & $3.87 \mathrm{E}-7 \pm 2.34 \mathrm{E}-7$ \\
\hline
\end{tabular}
gall ( $y$ ) and concentration of the bacterial antagonist Agrobacterium radiobacter $(z)$

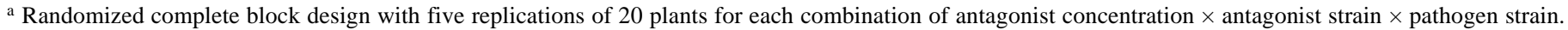
A. radiobacter concentrations are shown in Figure 2. Disease incidence was determined at 5 weeks after inoculation.

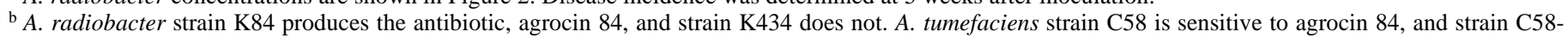
$\mathrm{R} 124$ is resistant to agrocin 84 .

c Initial parameter estimates obtained as described in text.

${ }^{\mathrm{d}}$ Final estimates for $k x, A$, and $c$, and their asymptotic standard errors were obtained by nonlinear regression (SAS NLIN secant method).

e Equation 6: $-\ln (1-y)=k x[(1-A)+A \cdot \exp (-c z)]$.

f Equation 3: $y=1-\exp (-k x\{[1-A]+A[\exp (-c z)]\})$.

${ }^{\mathrm{g}} \mathrm{H}_{\mathrm{o}}: c=0.0$ rejected at $P=0.05$.

${ }^{\text {h }} \mathrm{H}_{\mathrm{o}}: A=1.0$ rejected at $P=0.05$.

i Nonlinear regression procedure failed to converge. 
or C58-R124; $P \leq 0.05)$. The null hypothesis that $c=0.0$ was rejected $(P \leq 0.05)$ only for models based on the strain combination of K84 with C58 (Table 1). Statistically, models for the strain combination of K84 with C58 varied from models for the other strain combinations only by significant differences $(P \leq 0.05)$ in the estimates of $A$. Graphically, crown gall incidence plotted against antagonist concentration also showed the asymptotic nature of the doseresponse function when activity of agrocin 84 was muted by lack of agrocin 84 production in $A$. radiobacter or by resistance to agrocin 84 in A. tumefaciens (Figs. 2 and 3).

Cherry experiment. Incidence of crown gall on cherry seedlings inoculated with only $A$. tumefaciens at a concentration of $1 \times$ $10^{7}$ or $1 \times 10^{8} \mathrm{CFU} / \mathrm{ml}$ averaged $56 \pm 5$ and $65 \pm 7 \%$, respectively, for strain $\mathrm{K} 27 \mathrm{Rf}^{\mathrm{r}}$, and $45 \pm 3$ and $67 \pm 5 \%$, respectively, for strain $\mathrm{B} 49 \mathrm{c} \mathrm{Rf}$. Disease incidence on noninoculated control plants that had been root-pruned and planted in the same field averaged $2 \pm$ $1 \%$, indicating a low level of infection by naturally occurring strains of $A$. tumefaciens. At high concentrations of K84 $(\geq 2.5 \times$ $10^{8} \mathrm{CFU} / \mathrm{ml}$ ), incidence of plants galled by the agrocin 84 -sensitive strain $\mathrm{K} 27 \mathrm{Rf}^{\mathrm{T}}$ averaged $12 \pm 3 \%$ at the lower pathogen concentration and $26 \pm 5 \%$ at the higher concentration. In contrast, incidence of galled plants pretreated with a high concentration of K84 followed by inoculation with the agrocin 84-resistant strain $\mathrm{B} 49 \mathrm{c} \mathrm{Rf^{ \textrm {T } }}$ averaged $27 \pm 5$ and $54 \pm 7 \%$ for the low and high concentrations of the pathogen, respectively.

For $\mathrm{K} 27 \mathrm{Rf}^{\mathrm{r}}$, the initial estimates of the refuge parameter, $A$, were 0.86 and 0.79 for the pathogen concentrations of $1 \times 10^{7}$ and $1 \times 10^{8} \mathrm{CFU} / \mathrm{ml}$, respectively (Table 2 ). Conversely, initial estimates of $A$ were 0.51 and 0.32 for the low and high concentrations, respectively, of B49c $\mathrm{Rf}^{\mathrm{T}}$. For all strain-dose combinations, initial values of $c$ ranged from $4.85 \times 10^{-9}$ to $1.34 \times 10^{-8}$. Models were fit to data from all combinations of strains and concentrations (Table 2 ). For models based on either equation 3 or 6 , final estimates of $k x$ and $A$ varied from initial estimates by $<12 \%$, but final estimates of $c$ varied by up to a $\log$ unit from the initial value.

As with the tomato experiments, the derived dose-response models appeared to describe the observed data based on evaluation of graphical arrays (Fig. 4) and residual patterns (data not shown). The null hypothesis that $A=1.0$ was rejected $(P \leq 0.05)$ for each data set, except for the equation 6 model based on $\mathrm{K} 27 \mathrm{Rf}^{\mathrm{r}}$ at $1 \times$ $10^{8} \mathrm{CFU} / \mathrm{ml}$. The null hypothesis that $c=0.0$ was rejected $(P \leq$ $0.05)$ for three of four models based on the agrocin 84-sensitive strain K27 $\mathrm{Rf}^{\mathrm{r}}$ but not for the agrocin 84-resistant strain B49c $\mathrm{Rf}^{\mathrm{T}}$. Statistically, at similar initial concentrations of the pathogen, estimates of $A$ for strain $\mathrm{K} 27 \mathrm{Rf}^{\mathrm{T}}$ were significantly larger $(P \leq$ $0.05)$ than those obtained for strain $\mathrm{B} 49 \mathrm{c} \mathrm{Rf}^{\mathrm{T}}$. Estimates of $c$ did not differ significantly $(P>0.05)$ among strain combinations.

\section{DISCUSSION}

As hypothesized, dose-response relationships for biological control of crown gall of tomato showed diminishing increases in disease suppression with increasing concentrations of the bacterial antagonist $A$. radiobacter. At high antagonist concentrations $(\geq 2.5 \times$ $10^{8} \mathrm{CFU} / \mathrm{ml}$ ), these relationships appeared to approach an asymptote at which additional increases in the antagonist concentration led to only minimal increases in disease suppression.

Also as hypothesized, the expectation that the antibiotic agrocin 84 would be effective or ineffective for a tested strain combination was consistently reflected in the magnitude of the asymptotic limits. For example, for the combination of agrocin 84-producing K84 with agrocin 84-sensitive C58, the asymptote corresponded to a high level of disease control. Values of $A$ for this combination ranged from 0.92 to 0.95 , which indicates that 92 to $95 \%$ effective pathogen propagules were susceptible to a high concentration of K84. In contrast, use of either an agrocin 84-deficient strain of A. radiobacter or an agrocin 84-resistant strain of $A$. tumefaciens resulted in estimates of $A$ that ranged from 0.25 to 0.56 , indicating that 44 to $75 \%$ effective pathogen propagules were not susceptible to the antagonist when the activity of agrocin 84 had been muted. Estimates of $A$ were the only parameter that differed significantly among the nonlinear regression models based on strain combinations with either operative or muted agrocin 84 activity. Compared with models based on K84 with C58, however, the nonlinear regression models based on strain combinations with muted activity of agrocin 84 had relatively larger standard errors for estimates of the efficiency parameter, $c$. Thus, the null hypothesis that $c=0.0$ was not rejected for any of the models based on K84 with C58-R124, or K434 with C58 or C58-R124, whereas $c$ was concluded to be significantly greater than zero for models based on the combination of K84 with C58.
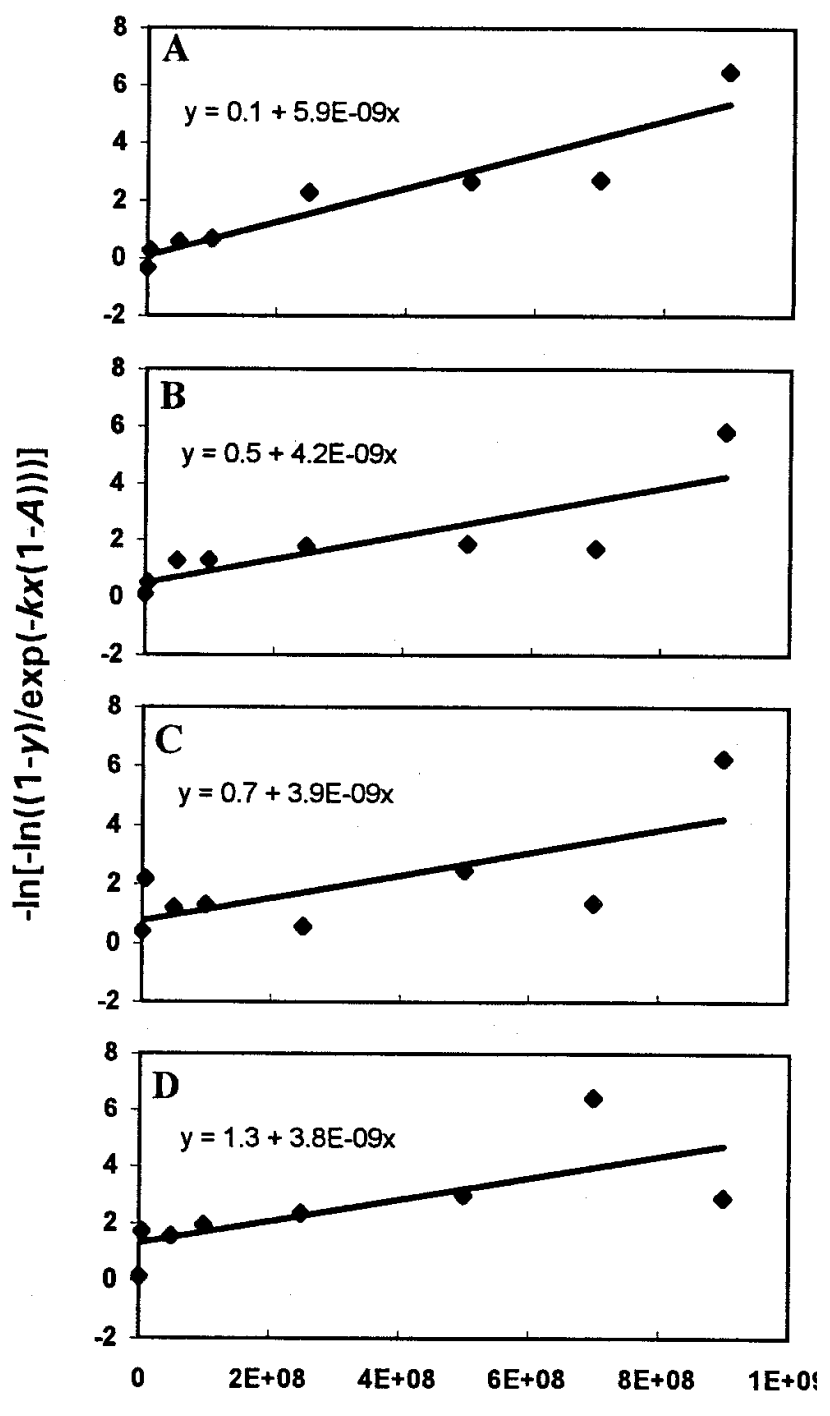

Initial antagonist concentration

Fig. 1. Linear regression of the incidence of $\mathrm{cv}$. Bonny Best tomato plants with crown gall $(y)$ on concentration of the biological control agent Agrobacterium radiobacter. Incidence data were transformed $-\ln (-\ln \{[1-y] /$ $\exp [-k x(1-A)]\})$, in which $k x$ and $A$ were initial estimates of parameters representing the relative efficiency of $A$. tumefaciens inoculum and the maximum proportion of effective pathogen units that can be rendered ineffective by $A$. radiobacter, respectively. Values for $k x$ and $A$ were obtained as described in text. The slope of the line, $c$, is an estimate of the average efficiency of an A. radiobacter CFU. A, Combination of agrocin 84-producing A. radiobacter strain K84 with agrocin 84-sensitive A. tumefaciens strain C58 $\left(r^{2}=\right.$ $0.87 ; \mathrm{H}_{\mathrm{o}}: c=0.0$ rejected at $\left.P=0.01\right)$. B, Combination of agrocin 84deficient $A$. radiobacter strain $\mathrm{K} 434$ with strain C58 $\left(r^{2}=0.69 ; \mathrm{H}_{\mathrm{o}}: c=0.0\right.$ rejected at $P=0.01)$. C, Combination of strain $\mathrm{K} 84$ with agrocin 84-resistant A. tumefaciens strain C58-R124 $\left(r^{2}=0.51 ; \mathrm{H}_{\mathrm{o}}: c=0.0\right.$ rejected at $\left.P=0.05\right)$. D, Combination of strain K434 with strain C58-R124 $\left(r^{2}=0.52 ; \mathrm{H}_{\mathrm{o}}: c=0.0\right.$ rejected at $P=0.05)$. Data are from tomato experiment 2 . 
For cherry, the nonlinear regression models that resulted to describe the effects of $\mathrm{K} 84$ concentration on crown gall response were similar to those observed in tomato. Estimates of $A$ were significantly larger for the strain combination in which agrocin 84 was expected to be effective (K84 with $\mathrm{K} 27 \mathrm{Rf}^{\mathrm{r}}$ ) compared with the combination in which it was expected to be ineffective (K84 with

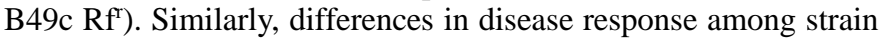
combinations used on cherry were not consistently reflected in estimates of $c$; although, owing to the relative size of the asymptotic standard errors, estimates of this parameter were significantly greater than zero for three of four models based on the combination of $\mathrm{K} 84$ with $\mathrm{K} 27 \mathrm{Rf}^{\mathrm{T}}$ but not different from zero for the combination of $\mathrm{K} 84$ with $\mathrm{B} 49 \mathrm{c} \mathrm{Rf}{ }^{\mathrm{T}}$. In contrast to the tomato data, the cherry experiment resulted in somewhat smaller estimates of $A$ for the treatment representing K84 combined with a pathogen strain sensitive to agrocin 84 . This result may indicate that either the host environment or the pathogen strains are additional factors that influence the asymptotic level of disease suppression. The pathogen strains differed among tomato and cherry experiments because C58 and its derivative are only weakly virulent on cherry (L. Moore, personal communication).

When analyzing the data from these experiments, we attempted to develop an objective and thorough process for estimation of the model parameters. Following procedures outlined previously (9), first estimates of $k x, A$, and $c$ were obtained that were then used to provide final estimates based on the iterative, nonlinear regression of two different forms of the model. Final estimates of $k x$ and $A$ were generally within $20 \%$ of the initial estimates, but final esti- mates of $c$ varied up to two $\log$ units from the initial estimates and were estimated with relatively poor precision (large values for the asymptotic standard error). Interestingly, among the strain combinations used on each host, the initial estimates of $c$ derived by the linear regression were relatively similar to each other, ranging from $1.5 \times 10^{-9}$ to $7.5 \times 10^{-9}$ on tomato and $4.9 \times 10^{-9}$ to $1.2 \times 10^{-8}$ on cherry. Moreover, estimates of $c$ derived by linear regression were, in most cases, found to be significantly greater than zero (Fig. 1). In sensitivity analyses, we found that estimates of $c$ derived by nonlinear regression methods were influenced greatly by the value of data points at low antagonist concentrations (e.g., $<1 \times$ $10^{6} \mathrm{CFU} / \mathrm{ml}$ for the experiments reported here) but were relatively insensitive to the position of data points at higher antagonist concentrations. Montesinos and Bonaterra (15), in a similar analysis of dose-response data, also noted difficulty obtaining precise estimates of parameters analogous to $c$. As suggested by these authors, the precision of estimates of $c$ obtained by nonlinear regression techniques might have been improved in our experiments if the replication of the zero and lower antagonist doses had been increased several-fold within the experimental design. Alternatively, linear regression also can be used to evaluate the significance of $c$, although it is apparent that the estimated values of this parameter will vary among the regression methods. In spite of these shortcomings, comparison among the forms of the model fitted to data (as well as sensitivity analyses we have conducted) indicates that estimates of $A$ were relatively independent of values of $c$. This observation adds support to the conclusion that $A$ is a biologically important parameter that is useful for characterizing the differences
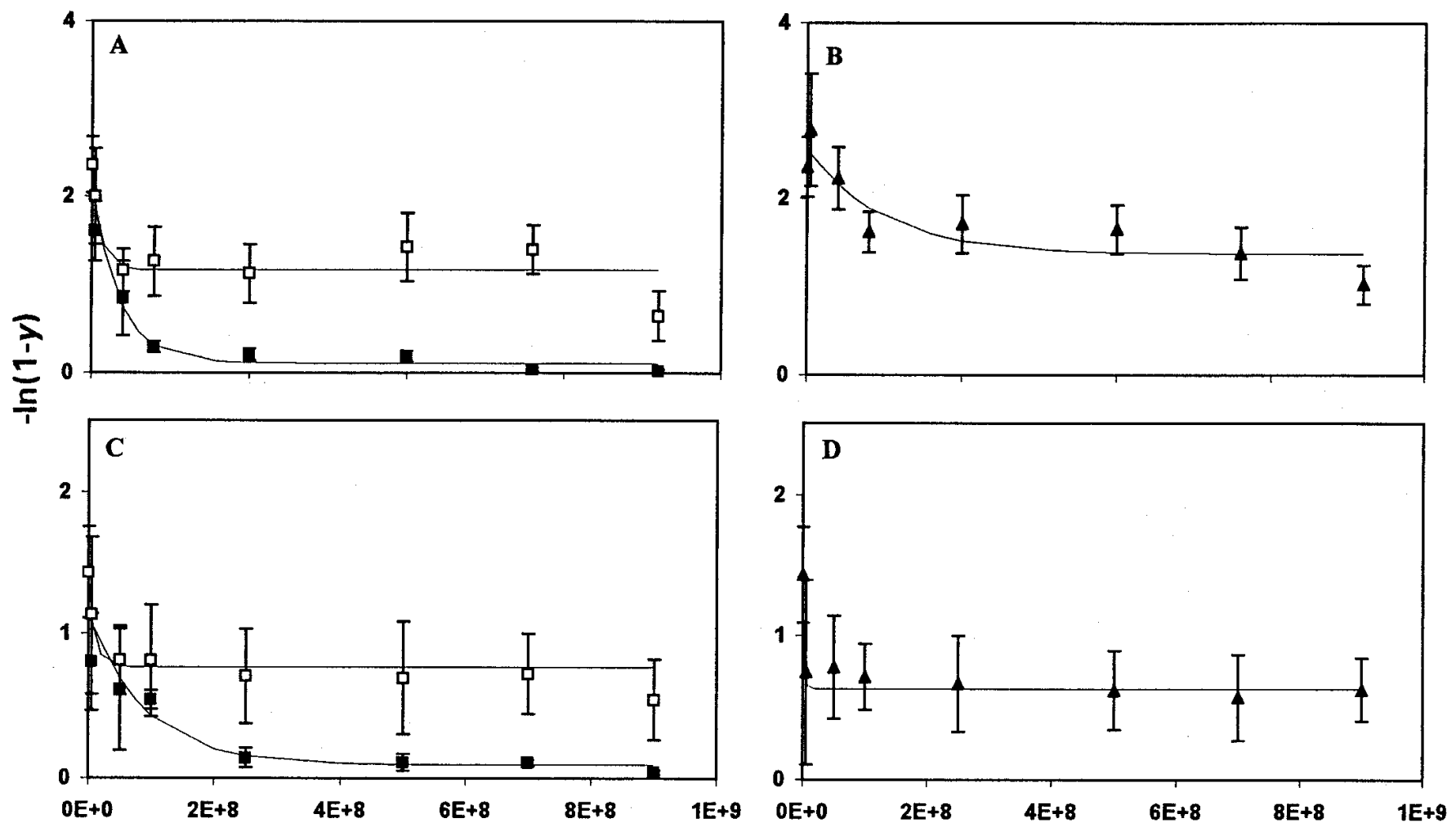

\section{Initial antagonist concentration}

Fig. 2. Incidence of cv. Bonny Best tomato plants with crown gall ( $y$ ) as affected by the concentration of Agrobacterium radiobacter applied prior to inoculation with the pathogen A. tumefaciens. Data points represent incidence of disease (transformed to correct for multiple infection, $-\ln [1-y]$ ), and the regression lines are based on equation $6,-\ln (1-y)=k x[(1-A)+A \exp (-c z)]$, in which $k x$ represents the relative efficiency of $A$. tumefaciens inoculum, $A$ represents the maximum proportion of effective pathogen units that can be rendered ineffective by A. radiobacter, $c$ is the average efficiency of an $A$. radiobacter CFU, and $z$ is the initial concentration of $A$. radiobacter $(\mathrm{CFU} / \mathrm{ml})$. A (experiment 1$)$ and $\mathbf{C}$ (experiment 2), Treatment with the agrocin 84-producing A. radiobacter strain $\mathrm{K} 84$ followed by inoculation with agrocin 84-sensitive A. tumefaciens strain C58 ( $)$ or treatment with the agrocin 84-deficient A. radiobacter strain K434 followed by inoculation with C58 $(\square)$. B (experiment 1) and D (experiment 2), Treatment with strain K434 followed by inoculation with agrocin 84-resistant $A$. tumefaciens strain C58-R124 ( $\mathbf{\Delta})$. The line drawn through each data point represents \pm one standard error of the mean. Parameter estimates for the regression lines are shown in Table 1. 
in efficacy among antagonist strains. Conversely, the efficiency parameter, $c$, is less useful for comparing strains, in part because it is difficult to estimate with high precision. More fundamentally, $c$ is less useful for comparing antagonist strains because differences in this parameter can be compensated for by adjusting the dose $(z)$, which is not true for the asymptotic differences reflected in values of $A(9)$.

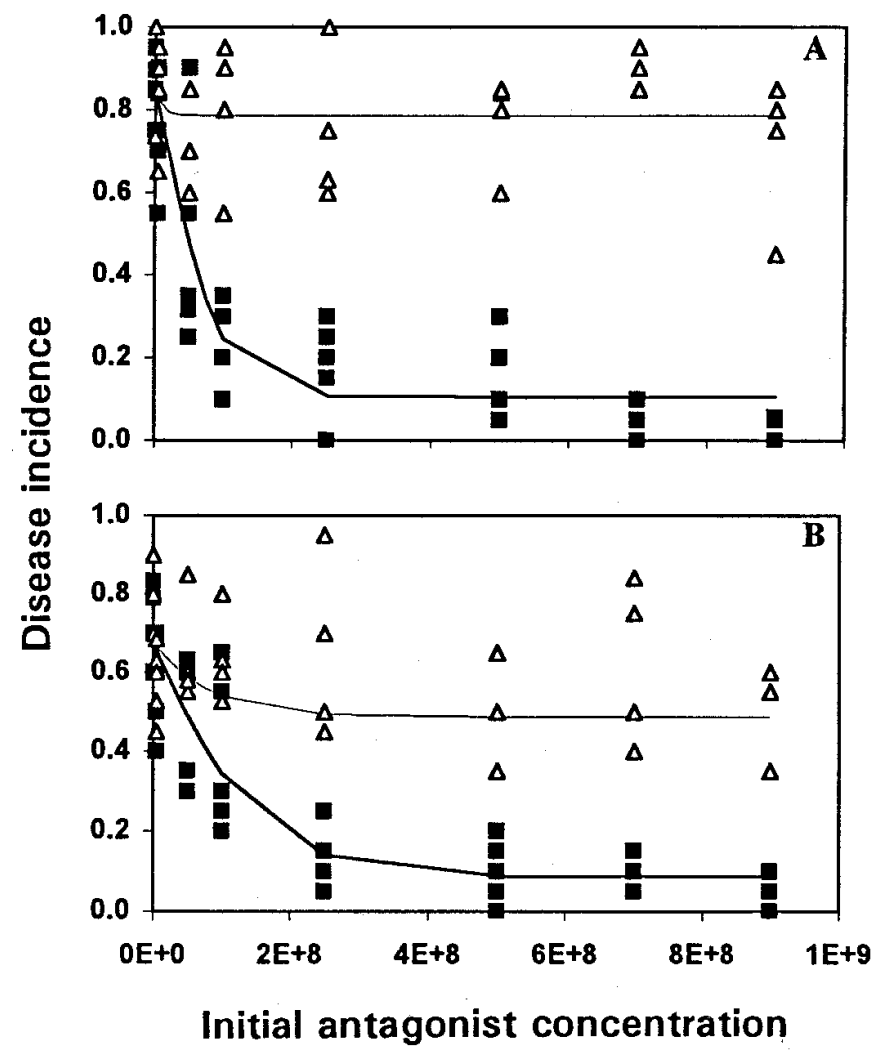

Fig. 3. Incidence of cv. Bonny Best tomato plants with crown gall as affected by the concentration of Agrobacterium radiobacter applied prior to inoculation with the pathogen A. tumefaciens. Data points represent incidence of disease $(y)$, and the regression line is based on equation $3, y=1-\exp \{-k x[(1-A)+$ $A \cdot \exp (-c z)]\}$, in which $k x$ represents the relative efficiency of $A$. tumefaciens inoculum, $A$ represents the maximum proportion of effective pathogen units that can be rendered ineffective by A. radiobacter, $c$ is the average efficiency of an $A$. radiobacter $\mathrm{CFU}$, and $z$ is the initial concentration of $A$. radiobacter $(\mathrm{CFU} / \mathrm{ml})$. $=$ Treatment with the agrocin 84-producing $A$. radiobacter strain K84 followed by inoculation with agrocin 84-sensitive A. tumefaciens strain C58. $\Delta=$ Treatment with strain $\mathrm{K} 84$ followed by inoculation with agrocin 84-resistant A. tumefaciens strain C58-R124. A, Experiment 1. B, Experiment 2. Each data point represents an experimental unit of 20 plants. Parameter estimates for the regression lines are shown in Table 1.
That the mechanism of antibiosis can affect values of $A$ may take on added significance as values of this parameter become known for more biocontrol agents and systems. For example, for the few antagonist-pathogen strain combinations in which dose-response relationships have been modeled, the results indicate that effective and ineffective antagonist strains can be classified by their values

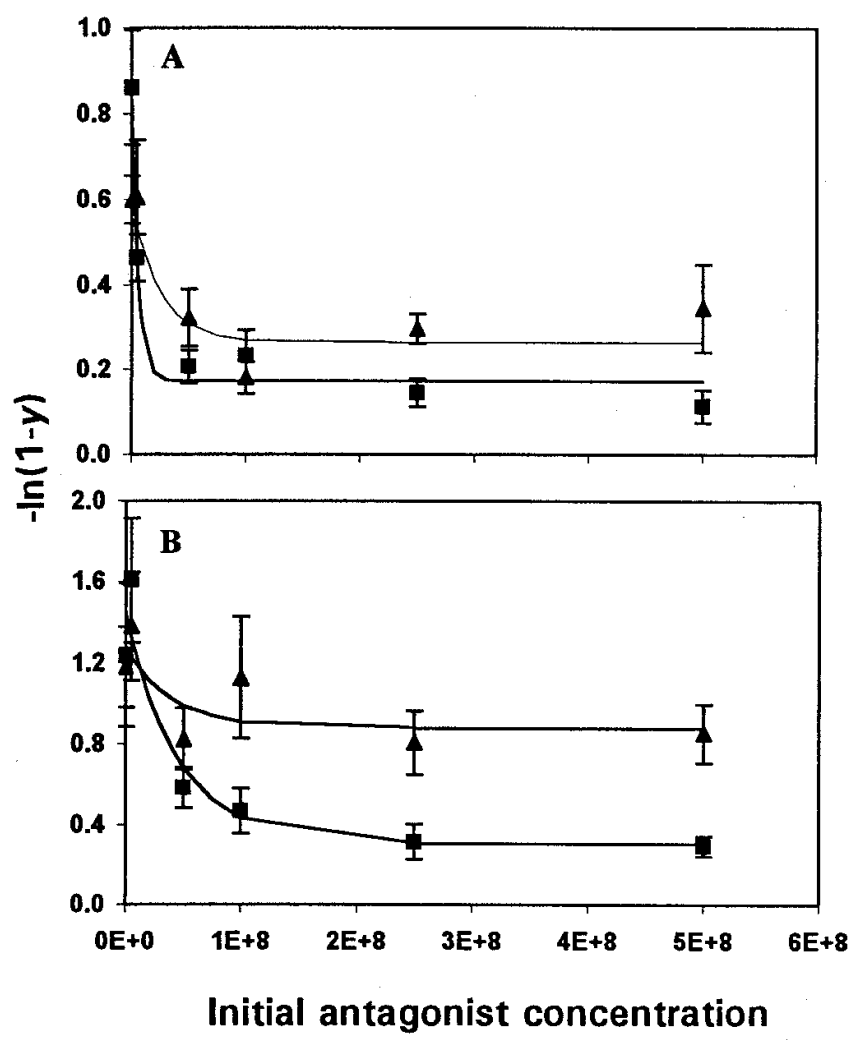

Fig. 4. Incidence of crown gall on field-grown mahaleb cherry seedlings $(y)$ as affected by the concentration of Agrobacterium radiobacter strain K84 applied prior to inoculation with the pathogen A. tumefaciens. Data points represent incidence of disease (transformed to correct for multiple infection, $-\ln [1-y])$, and the regression line is based on equation $6,-\ln (1-y)=k x[(1-A)+$ $A \cdot \exp (-c z)$ ], in which $k x$ represents the relative efficiency of $A$. tumefaciens inoculum, $A$ represents the maximum proportion of effective pathogen units that can be rendered ineffective by $A$. radiobacter, $c$ is the average efficiency of an $A$. radiobacter $\mathrm{CFU}$, and $z$ is the initial concentration of $A$. radiobacter (CFU). A, Inoculation with agrocin 84-sensitive A. tumefaciens strain K27 (而) or agrocin 84-resistant $A$. tumefaciens strain $\mathrm{B} 49 \mathrm{c}(\boldsymbol{\Lambda})$ at $1 \times 10^{7} \mathrm{CFU} / \mathrm{ml}$. B, Inoculation with strain $\mathrm{K} 27(\boldsymbol{\square})$ or strain B49c $(\boldsymbol{\Delta})$ at $1 \times 10^{8} \mathrm{CFU} / \mathrm{ml}$. The line drawn through each data point represents \pm one standard error of the mean. Parameter estimates for the regression lines are shown in Table 1.

TABLE 2. Parameter estimates for the dose-response equation used to describe the relationship between incidence of field-grown cherry seedlings ${ }^{\mathrm{a}}$ with crown gall $(y)$ and the concentration of the bacterial antagonist Agrobacterium radiobacter strain K84 (z)

\begin{tabular}{|c|c|c|c|c|c|c|c|c|c|c|}
\hline \multirow{2}{*}{$\begin{array}{l}\text { Pathogen } \\
\text { strain }^{\mathrm{b}}\end{array}$} & \multirow[b]{2}{*}{ Concentration } & \multicolumn{3}{|c|}{ Initial estimates $^{c}$} & \multicolumn{3}{|c|}{ Final estimates ${ }^{\mathrm{d}}$ for equation $6^{\mathrm{e}}$} & \multicolumn{3}{|c|}{ Final estimates for equation $3^{\mathrm{f}}$} \\
\hline & & $k x$ & $A$ & $c$ & $k x$ & $A$ & $c$ & $k x$ & $A$ & $c$ \\
\hline K27 & $1 \times 10^{7} \mathrm{CFU} / \mathrm{ml}$ & 0.86 & 0.87 & $1.20 \mathrm{E}-8$ & $86 \pm 0.05$ & $0.80 \pm 0.03 * g$ & $1.73 \mathrm{E}-7 \pm 4.23 \mathrm{E}-8 * * \mathrm{~h}$ & $0.83 \pm 0.08$ & $0.79 \pm 0.03^{*}$ & $1.65 \mathrm{E}-7 \pm 4.80 \mathrm{E}-8^{* *}$ \\
\hline B49c & $1 \times 10^{7} \mathrm{CFU} / \mathrm{ml}$ & 0.60 & 0.51 & E-9 & \pm 0.07 & \pm 0 & \pm 3 & $2 \pm$ & $7^{*}$ & $5 \mathrm{E}-8$ \\
\hline B $49 \mathrm{c}$ & $1 \times 10^{8} \mathrm{CFU} / \mathrm{ml}$ & 1.18 & 0.32 & $5.57 \mathrm{E}-9$ & $1.28 \pm 0.15$ & $0.31 \pm 0.12 *$ & $2.56 \mathrm{E}-8 \pm 3.86 \mathrm{E}-8$ & $1.20 \pm 0.17$ & $0.32 \pm 0.11^{*}$ & $4.57 \mathrm{E}-8 \pm 8.20 \mathrm{E}-8$ \\
\hline
\end{tabular}

a The experiment was conducted with 1-year-old seedlings of mahaleb cherry arranged in a randomized complete block design with six replications of 33 plants for each combination of antagonist concentration $\times$ pathogen strain $\times$ pathogen concentration. A. radiobacter concentrations are shown in Figure 4 . Seedlings were field planted in May and dug to assess disease in October.

${ }^{\mathrm{b}}$ A. tumefaciens strain $\mathrm{K} 27$ is sensitive to agrocin 84, the antibiotic produced by A. radiobacter strain K84, and A. tumefacians strain B49c is resistant to agrocin 84 .

${ }^{\mathrm{c}}$ Initial parameter estimates obtained as described in text.

${ }^{\mathrm{d}}$ Final estimates for $k x, A$, and $c$, and their asymptotic standard errors were obtained by nonlinear regression (SAS NLIN secant method).

e Equation 6: $-\ln (1-y)=k x[(1-A)+A \cdot \exp (-c z)]$.

${ }^{\mathrm{f}}$ Equation 3: $y=1-\exp (-k x\{[1-A]+A[\exp (-c z)]\})$.

$\mathrm{g}^{\mathrm{H}} \mathrm{H}_{\mathrm{o}}: A=1.0$ rejected at $P=0.05$.

${ }^{\mathrm{h}} \mathrm{H}_{\mathrm{o}}: c=0.0$ rejected at $P=0.05$. 
of $A$. Montesinos and Bonaterra (15) reported estimates of $A$ for four antagonist-pathogen combinations that ranged from 0.65 to 0.97 , with satisfactory disease control observed only with values of $A$ greater than 0.93. Similarly, Smith et al. (19) used dose-response models to assess the relative susceptibility of different tomato genotypes to damping-off by the fungus Pythium torulosum, in combination with an assessment of the ability of the same tomato genotypes to support the activity of bacterial antagonists of $P$. torulosum. Estimates of $A$ ranged from 0.0 to 0.94 for individual host genotype-antagonist strain combinations. Host genotypes that were good supporters of bacterial antagonists were characterized as having large values of $A(>0.86)$. In its broadest interpretation, values of $A$ show potential as a statistic by which the quality of an antagonist-pathogen-host interaction can be summarized. In other words, $A$ reflects that proportion of the pathogen population that is susceptible to an antagonist. Moreover, while this parameter is defined in experiments testing large doses of antagonist inoculum, it impacts the efficiency of disease suppression over the full range of antagonist densities (9).

The remarkable ability of strain K84 to suppress crown gall has been demonstrated in numerous studies reported over the last 25 years $(3,4,7,12,13,16,22)$. As in this study, others $(3,4,12,13)$ have demonstrated that agrocin 84 is important in this process by comparing the protection afforded by $\mathrm{K} 84$ with that achieved by K84-derived mutants that do not produce agrocin 84 , and by comparing the effect of K84 on incidence of crown gall caused by agrocin 84-sensitive and agrocin 84 -insensitive strains of the pathogen. Many reports $(7,13$, 14,22 ), however, have questioned whether activity of agrocin 84 (production of and sensitivity to) is essential for suppression. Donner et al. (5) and McClure et al. (14) have provided evidence that a second agrocin, agrocin 434, produced by both K84 and K434, is involved in biological control of crown gall incited by biovar 2 strains of $A$. tumefaciens. The data from this study provided some support for the hypothesis that mechanisms in addition to agrocin 84 may be involved in crown gall suppression. For example, for all strain combinations in which agrocin 84 activity was expected to be muted, high concentrations of K84 or K434 (>2.5 $\times 10^{8} \mathrm{CFU} / \mathrm{ml}$ for tomato and $>1 \times 10^{8} \mathrm{CFU} / \mathrm{ml}$ for cherry) averaged $27 \pm 3 \%$ less disease when compared with the no-antagonist control. Also, suppression of the agrocin-resistant strain B49c was greater when this strain was inoculated at $1 \times 10^{7} \mathrm{CFU} / \mathrm{ml}$ compared with $1 \times 10^{8} \mathrm{CFU} / \mathrm{ml}$ (Fig. 4), which is consistent with the observation that agrocin 84 is less important at low pathogen densities (7). Although our data do not provide insight on mechanisms operating for disease suppression other than agrocin 84, we suggest that the modeling approach taken in this study may be a powerful compliment to the genetic approach (14) in future research on this topic. In this regard, the modeling approach addresses some of Farrand and Wang's (7) recommendations related to the need to develop a definitive experimental framework within which the importance of antibiotics and other mechanisms of biocontrol can be assessed.

In summary, we conclude that the results obtained in this study supported our hypotheses. A dose-response model that was derived previously characterized the control of crown gall as a function of antagonist concentration. For modeled strain combinations, the estimated values of the asymptote (refuge) parameter were commonly less than 1.0, indicating that a portion of the A. tumefaciens inoculum was not susceptible to $A$. radiobacter, even at high concentrations of the antagonist. The differences among bacterial strain combinations in the magnitude of the asymptote defined by the dose-response relationships suggest that $A$. radiobacter impacts a reduced proportion of the pathogen population when the activity of agrocin 84 is muted. The results establish an epidemiological basis as to how the mechanism of antibiosis influences biological disease control.

\section{ACKNOWLEDGMENTS}

This is Oregon Agricultural Experiment Station Technical Paper No. 11,536. We thank T. Sawyer for technical assistance.

\section{LITERATURE CITED}

1. Baker, R. 1978. Inoculum potential. How disease develops in populations. Pages 137-157 in: Plant Disease: An Advanced Treatise, Vol. II. J. G. Horsfall and E. B. Cowling, eds. Academic Press, New York.

2. Campbell, C. L., and Madden, L. V. 1990. Introduction to Plant Disease Epidemiology. Wiley Interscience, New York.

3. Cooksey, D. A., and Moore, L. W. 1982. High frequency spontaneous mutations to agrocin resistance in Agrobacterium tumefacians and $A$. rhizogenes. Physiol. Plant Pathol. 20:129-135.

4. Cooksey, D. A., and Moore, L. W. 1982. Biological control of crown gall with an agrocin mutant of Agrobacterium radiobacter. Phytopathology 72:919-921.

5. Donner, S. C., Jones, D. A., McClure, N. C., Rosewarne, G. M., Tate, M. E., Kerr, A., Fajardo, N. N., and Clare, B. G. 1993. Agrocin 434, a new plasmid encoded agrocin from the biocontrol Agrobacterium strains K84 and K1026, which inhibits biovar 2 agrobacteria. Physiol. Mol. Plant Pathol. 42:185-194.

6. Farrand, S. K., Ryder, M. H., Hayman, G. T., O’Morchoe, S. B., Shim, J. S., and Kerr, A. 1987. Genetics and molecular biology of agrocin production and sensitivity in Agrobacterium. Curr. Plant Sci. Biotechnol. Agric. 4:42-55.

7. Farrand, S. K., and Wang, C. 1992. Do we really understand crown gall control by Agrobacterium radiobacter strain K84? Pages 287-293 in: Biological Control of Plant Diseases. E. C. Tjamos, G. C. Papavizas, and R. J. Cook, eds. Plenum Press, New York.

8. Gregory, P. H. 1948. The multiple infection transformation. Ann. Appl. Biol. 35:412-417.

9. Johnson, K. B. 1994. Dose-response relationships and inundative biological control. Phytopathology 84:780-784.

10. Johnson, K. B. 1998. Dose-response relationships in biocontrol of plant disease and their use to define pathogen refuge size. Pages 385-391 in: Theoretical Approaches to Biological Control. B. A. Hawkins and H. V. Cornell, eds. Cambridge University Press, Cambridge, United Kingdom.

11. Keane, P. J., Kerr, A., and New, P. B. 1970. Crown gall of stone fruits. II. Identification and nomenclature of Agrobacterium isolates. Aust. J. Biol. Sci. 23:585-595.

12. Kerr, A., and Htay, K. 1974. Biological control of crown gall through bacteriocin production. Physiol. Plant Pathol. 4:37-44.

13. Lopez, M. M., Gorris, M. T., Salcedo, C. I., Montojo, A. M., and Miro, M. 1989. Evidence of biological control of Agrobacterium tumefaciens sensitive and resistant to agrocin 84 by different Agrobacterium radiobacter strains on stone fruit trees. Appl. Environ. Microbiol. 55:741-746.

14. McClure, N. C., Ahmandi, A.-R., and Clare, B. R. 1998. Construction of a range of derivatives of the biological control strain Agrobacterium rhizogenes K84: Study of factors involved in the biological control of crown gall disease. Appl. Environ. Microbiol. 64:3977-3982.

15. Montesinos, E., and Bonaterra, A. 1996. Dose-response models in biological control of plant pathogens: An empirical verification. Phytopathology 86:464-472.

16. Moore, L. W., and Canfield, M. 1997. Biology of Agrobacterium and management of crown gall diseases. Pages 153-199 in: Management of Soilborne Plant Pathogens. R. Hall, ed. The American Phytopathological Society, St. Paul, MN.

17. Moore, L. W., and Warren, G. 1979. Agrobacterium radiobacter strain 84 and biological control of crown gall. Annu. Rev. Phytopathol. 17:163-179.

18. Raaijmakers, J. M., Leeman, M., van Oorschot, M. M. P., van der Sluis, I., Schippers, B., and Bakker, P. A. H. M. 1995. Dose-response relationships in biological control of Fusarium wilt of radish by Pseudomonas spp. Phytopathology 85:1075-1081.

19. Smith, K. P., Handelsman, J., and Goodman, R. M. 1997. Modeling doseresponse relationships in biological control: Partitioning host responses to the pathogen and biocontrol agent. Phytopathology 87:720-729.

20. Stockwell, V. O., Moore, L. W., and Loper, J. E. 1993. Fate of Agrobacterium radiobacter $\mathrm{K} 84$ in the environment. Appl. Environ. Microbiol. 59:2112-2120.

21. van der Plank, J. E. 1975. Principles of Plant Infection. Academic Press, New York.

22. Vicedo, B., Penalver, R., Asins, M. J., and Lopez, M. M. 1993. Biological control of Agrobacterium tumefaciens, colonization, and pAgK84 transfer with Agrobacterium radiobacter $\mathrm{K} 84$ and the tra ${ }^{-}$mutant strain K1026. Appl. Environ. Microbiol. 59:309-315. 